phys. stat. sol. (a) 190, No. 1, 181-186 (2002)

\title{
Polariton Lasing Due to the Exciton-Electron Scattering in Semiconductor Microcavities
}

\author{
G. Malpuech ${ }^{1}$ ) (a), A. Kavokin (b), A. Di Carlo (a), J. J. Baumberg (c), \\ F. Compagnone (a), P. Lugli (a), and M. Zamfirescu (b)
}

(a) INFM - Dept. Elect. Eng., University of Rome "Tor Vergata",

Via di Tor Vergata 110, I-00133 Roma, Italy

(b) LASMEA, CNRS - Université Clermont-II "Blaise Pascal", 24 Avenue des Landais, F-63177 Aubiere Cedex, France

(c) Department of Physics and Astronomy, University of Southampton, SO17 1BJ, UK

(Received September 26, 2001; accepted October 31, 2001)

Subject classification: 42.55.Sa; 71.36.+c; 78.47.+p; S7.12

The issue of polariton lasing in n-doped microcavities is addressed. We show theoretically that the introduction of a cold electron gas into quantum microcavities induces efficient electron-polariton scattering. This process allows the condensation of the polaritons accumulated at the bottleneck to the final emitting state with a transition time of a few picoseconds, opening the way to a new generation of low-threshold light emitting devices.

Achievement of polariton lasing at room temperature has become a major challenge for the semiconductor physicists of today. It requires new materials and new physical principles. GaN, showing stable excitons at room temperature, is an excellent candidate for being a basic material of a new generation of semiconductor lasers [1]. The fundamental problem to overcome is the bottleneck effect, i.e. kinetic blocking of the polariton relaxation down to the ground state that prevents the Bose condensation of polaritons necessary for polariton lasing. Here we propose a new polariton relaxation mechanism in a microcavity that is based on the scattering of polaritons with free electrons. This allows polaritons to efficiently relax from the bottleneck [2-4] region to the lowest energy state once free electrons are introduced into the active region either via doping or by photo-excitation. This opens the way to realization of low-threshold laserlike devices based on cavity polaritons.

The basic principle of a polariton laser is illustrated in Fig. 1a using the dispersion curve of the lower branch exciton-polaritons in a typical microcavity. The strong coupling regime creates a trap containing a small number of polariton states at energies below all other states in the semiconductor. This polariton trap is sharp with a depth equal to nearly half the splitting $\Omega$ between the two polariton modes. Polaritons in the trap are half photon and half exciton and have properties suitable for the Bose condensation of exciton-polaritons once sufficiently populated. Recombination from this state in the Bose condensation regime is coherent and monochromatic, characteristic of laser emission. The relaxation of polaritons into the $k=0$ state is found to be stimulated if the population of the final state is larger than unity [5].

The major problem to be resolved on the way towards realization of polariton lasers comes from a relaxation bottleneck $[2,3]$ in the dispersion relation. For small in-plane

\footnotetext{
1) Corresponding author; e-mail: malpuech@lasmea.univ-bpclermont.fr
} 
(a)

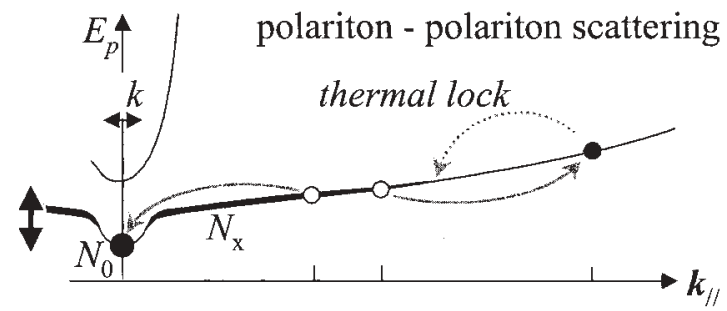

(b)

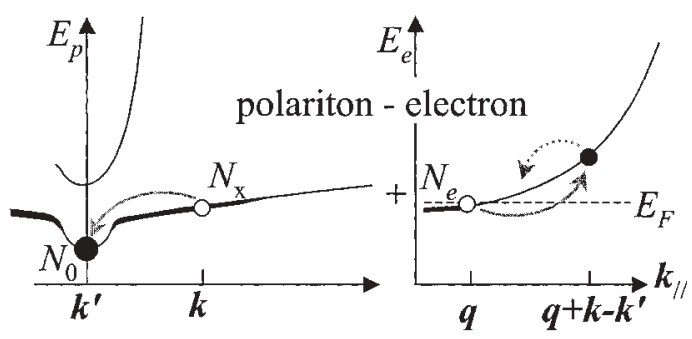

Fig. 1. a) Dispersion relations of upper and lower polaritons, showing the dominant pair scattering of polaritons feeding energy into the trap at $k=0$. b) Similar polariton-electron scattering process. The excited carriers quickly relax, preventing re-ionization

wave vectors, acoustic phonons cannot assist in the polariton relaxation, and polaritons accumulate in a broad bottleneck region. Nevertheless, in recent years new experimental results have appeared giving hope for realization of polariton lasers [7, 8]. As we will show, this strong luminescence originates from the trap states thanks to polaritonpolariton scattering processes (Fig. 1a). Under resonant excitation, the high efficiency of pair scattering processes between polaritons has been shown under pulsed excitation $[5,9]$, cw excitation [10], and theoretically [11]. These results clearly demonstrate that cavity polaritons behave as bosons rather than fermions and that they can condense in the trap if a resonant scattering process is available. However, polariton-polariton scattering is only efficient for resonant optical pumping of the cavity. The exciton-exciton scattering is too weak to allow the polariton trap population to exceed unity [3, 4]. Although polariton-polariton scattering is promising for realising optical devices such as parametric oscillators, it is unsuitable for polariton lasers. Here we show that if a concentration of free electrons is introduced into the structure, polaritons can be efficiently scattered from the bottleneck region to the ground state (Fig. 1b). Through this process, a polariton passes its excess energy and wave vector to a free electron, as described by

$$
\begin{aligned}
& \mathrm{e}^{-}(\mathbf{q})+\mathrm{P}(\mathbf{k}) \rightarrow \mathrm{e}^{-}\left(\mathbf{q}+\mathbf{k}-\mathbf{k}^{\prime}\right)+\mathrm{P}\left(\mathbf{k}^{\prime}\right), \\
& E\left(\mathbf{k}^{\prime}\right)+\frac{\hbar^{2}\left(\mathbf{q}+\mathbf{k}-\mathbf{k}^{\prime}\right)^{2}}{2 m_{\mathrm{e}}}-\frac{\hbar^{2} \mathbf{q}^{2}}{2 m_{\mathrm{e}}}=E(\mathbf{k}),
\end{aligned}
$$

where $\mathrm{e}^{-}$denotes an electron, $E$ is the polariton energy, and $m_{\mathrm{e}}$ is the electron effective mass. The polaritons cannot be re-ionized because the excited electrons rapidly thermalise with the electron distribution due to the electron-electron scattering that we expect to be extremely efficient, providing a 'thermal lock' for the trapped polaritons. The advantage of this scattering mechanism with respect to previously discussed ones is that (i) the matrix element of electron-exciton scattering is quite large [13], and (ii) the electron has a much lighter mass than an exciton, thus the energy relaxation of a polariton from the bottleneck region to the ground state requires fewer scattering events or 
a lower carrier density than for polariton-polariton or polariton-phonon scattering. Note also that we neglect the trions in the present model. In case of trion formation, collisions with free electrons may cause the resonant dissociation of trions, that might be stimulated. This process will be considered in detail in our further publications.

We simulate the relaxation of cavity polaritons excited non-resonantly in a steadystate regime. A Boltzmann equation is solved numerically in two-dimensional reciprocal space,

$$
\frac{\mathrm{d} n_{k}}{\mathrm{~d} t}=P_{k}-\Gamma_{k} n_{k}-n_{k} \sum_{\mathbf{k}^{\prime}} W_{\mathbf{k} \rightarrow \mathbf{k}^{\prime}}\left(n_{k^{\prime}}+1\right)+\left(n_{k}+1\right) \sum_{\mathbf{k}^{\prime}} W_{\mathbf{k}^{\prime} \rightarrow \mathbf{k}} n_{k^{\prime}},
$$

where $n_{k}$ is the polariton distribution function in the state having a wave vector $\mathbf{k}, P_{k}$ is the generation term describing optical pumping, $\Gamma_{k}$ is the recombination rate composed of both radiative and non-radiative components, and $W_{\mathbf{k} \rightarrow \mathbf{k}^{\prime}}$ is the total scattering rate between the states $\mathbf{k}$ and $\mathbf{k}^{\prime}$. This scattering rate is comprised of three terms in our model, namely, polariton-acoustic phonon scattering (rate $W_{\mathrm{a}}$ ), polariton-polariton scattering (rate $W_{\mathrm{x}}$ ), and polariton-electron scattering (rate $W_{\mathrm{e}}$ ). Only the intra-branch scattering within the lower polariton branch is considered. The cavity photon lifetime $\tau_{\mathrm{c}}$ is taken to be $8 \mathrm{ps}$, whereas the radiative life time of the QW excitons is taken to be 20 ps (typical for InGaAs QWs). The polariton non-radiative lifetime is chosen constant over the whole reciprocal space $(1 \mathrm{~ns})$. The polariton-acoustic phonon and the polariton-polariton scattering rates are calculated as in [2]. The polariton-electron scattering rate is calculated using the Fermi golden rule as

$$
\begin{aligned}
W_{\mathbf{k} \rightarrow \mathbf{k}^{\prime}}^{\mathrm{e}}= & \frac{2 \pi}{\hbar} \sum_{\mathbf{q}} M^{2} N_{\mathbf{q}}\left(1-N_{\mathbf{q}+\mathbf{k}^{\prime}-\mathbf{k}}\right) \\
& \times \delta\left[E\left(\mathbf{k}^{\prime}\right)-E(\mathbf{k})-\frac{\hbar^{2}}{2 m_{\mathrm{e}}}\left(\mathbf{q}^{2}-\left|\mathbf{q}+\mathbf{k}-\mathbf{k}^{\prime}\right|^{2}\right)\right],
\end{aligned}
$$

where $\mathbf{q}$ is the wave vector of an electron required for the resonant scattering and $N_{\mathbf{q}}$ is the Fermi-Dirac electron distribution function. The electron distribution is not strongly perturbed by the exciton-electron scattering in the low-pumping regime we consider. Only a small part of electrons is accelerated by scattering with excitons. Thus we assume the equilibrium Fermi-distribution for the electron gas. Initially, an electron concentration $n_{\mathrm{e}}=10^{10} \mathrm{~cm}^{-2}$ and a temperature of $10 \mathrm{~K}$ are assumed. The exciton screening is intrinsically taken into account in the calculated polariton dispersion curve $E(\mathbf{k}) . M$ is the matrix element of interaction between an electron and a polariton. In the Born approximation, $M=M_{\mathrm{dir}} \pm M_{\mathrm{exc}}$, corresponding to the triplet configuration ( + , parallel electron spins) and the singlet configuration (-, antiparallel electron spins). Further, we shall only include the stronger electron-polariton scattering of the triplet configuration.

$$
\begin{aligned}
& M_{\mathrm{dir}}=\frac{2 \pi e^{2}}{A \varepsilon} \frac{x_{k} x_{k^{\prime}}}{\left|\mathbf{k}-\mathbf{k}^{\prime}\right|}\left[\left(1+\xi_{\mathrm{h}}^{2}\right)^{-\frac{3}{2}}-\left(1+\xi_{\mathrm{e}}^{2}\right)^{-\frac{3}{2}}\right], \\
& M_{\mathrm{exc}}=\frac{8 \pi e^{2}}{A \varepsilon} \frac{x_{k} x_{k^{\prime}}}{\left(a^{-2}+\left|\mathbf{q}-\beta_{\mathrm{e}} \mathbf{k}^{\prime}\right|^{2}\right)^{1 / 2}}\left[\left(1+\xi_{\mathrm{c}}^{2}\right)^{-\frac{3}{2}}-\left(1+4 \xi_{\mathrm{h}}^{2}\right)^{-\frac{3}{2}}\right],
\end{aligned}
$$

where $\varepsilon$ is the dielectric susceptibility of the $\mathrm{QW}, x_{k}$ is the exciton fraction of the polariton state having a wave vector $\mathbf{k}, \zeta_{\mathrm{e}, \mathrm{h}}=(1 / 2) \beta_{\mathrm{e}, \mathrm{h}}\left|\mathbf{k}-\mathbf{k}^{\prime}\right| a, \zeta_{\mathrm{c}}=\left(\beta_{\mathrm{e}} \mathbf{k}+\left|\mathbf{k}-\mathbf{k}^{\prime}\right|-\mathbf{q}\right) a$, 


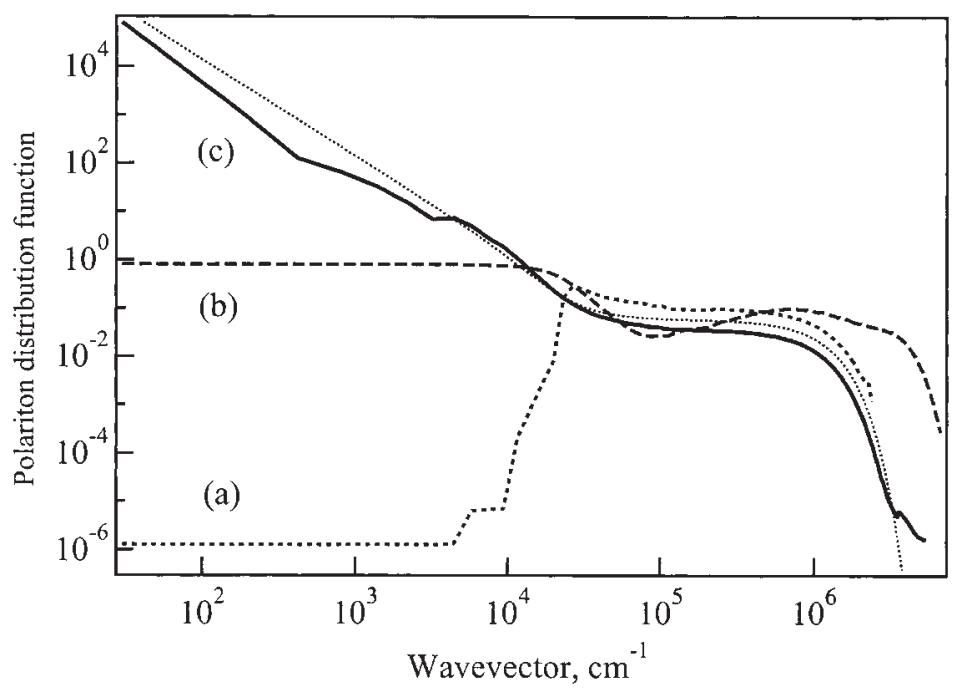

Fig. 2. Distribution function of polaritons at $10 \mathrm{~K}$ when non-resonantly pumped with a power of $4.2 \mathrm{~W} / \mathrm{cm}^{2}$. Results are shown for (a) polariton-acoustic phonon scattering (dotted), (b) as (a) plus polariton-polariton scattering (dashed), and (c) as (b) plus polariton-electron scattering (solid). The thin dotted line shows the equilibrium Bose distribution function with zero chemical potential

$\beta_{\mathrm{e}, \mathrm{h}}=m_{\mathrm{e}, \mathrm{h}} /\left(m_{\mathrm{e}}+m_{\mathrm{h}}\right)$, where $m_{\mathrm{e}}$ and $m_{\mathrm{h}}$ are electron and hole effective masses, respectively, $a$ is the in-plane Bohr radius of the QW exciton, and $A$ is a normalisation area.

Figure 2 shows the equilibrium distribution function under continuous pumping for a cavity having a normal-mode splitting of $5 \mathrm{meV}$ containing a single QW, and for zero detuning of cavity and exciton modes. For all the curves the pump power absorbed by the single QW is set to be $4.2 \mathrm{~W} / \mathrm{cm}^{2}$ between $k=3 \times 10^{6}$ and $5 \times 10^{6} \mathrm{~cm}^{-1}$, roughly equivalent to an excess of energy of $20 \mathrm{meV}$. Taking into account only the acoustic phonon scattering (curve a in Fig. 2), a thermalised population is seen only beyond $k=2 \times 10^{4} \mathrm{~cm}^{-1}$ (the bottleneck region) where the polaritons accumulate. Equilibrium is reached after $10 \mathrm{~ns}$ leaving a total polariton population of $2.5 \times 10^{10} \mathrm{~cm}^{-2}$. Including both polariton-polariton and polariton-acoustic phonon scattering processes (curve $b$ in Fig. 2) shows partial relaxation of the bottleneck and a flat polariton distribution. However, the equilibrium polariton density in the cavity remains the same, close to the saturation density for excitons $\left(\approx 5 \times 10^{10} \mathrm{~cm}^{-2}\right)$. The population of the ground state is close to unity. This result is in excellent agreement with experimental results obtained up to now [4], which shows that the threshold amplification for the population of the trap state is reached when the strong coupling regime is likely to be destroyed.

When all three scattering processes listed above are taken into account (curve $c$ in Fig. 2), a huge occupation number of the lowest energy state of more than $10^{4}$ is achieved. This system thus acts as a polariton laser in that the scattering of polaritons injected at high $k$ by optical or electrical pumping is stimulated by the population of the low $k$-states. In this situation the light power emitted in a cone of $1^{\circ}$ is $3.3 \mathrm{~W} / \mathrm{cm}^{2}$ and the efficiency of the energy transfer from pump to emitted light is about $80 \%$. The light emitted by the cavity is much more directional and comes from a smaller number 

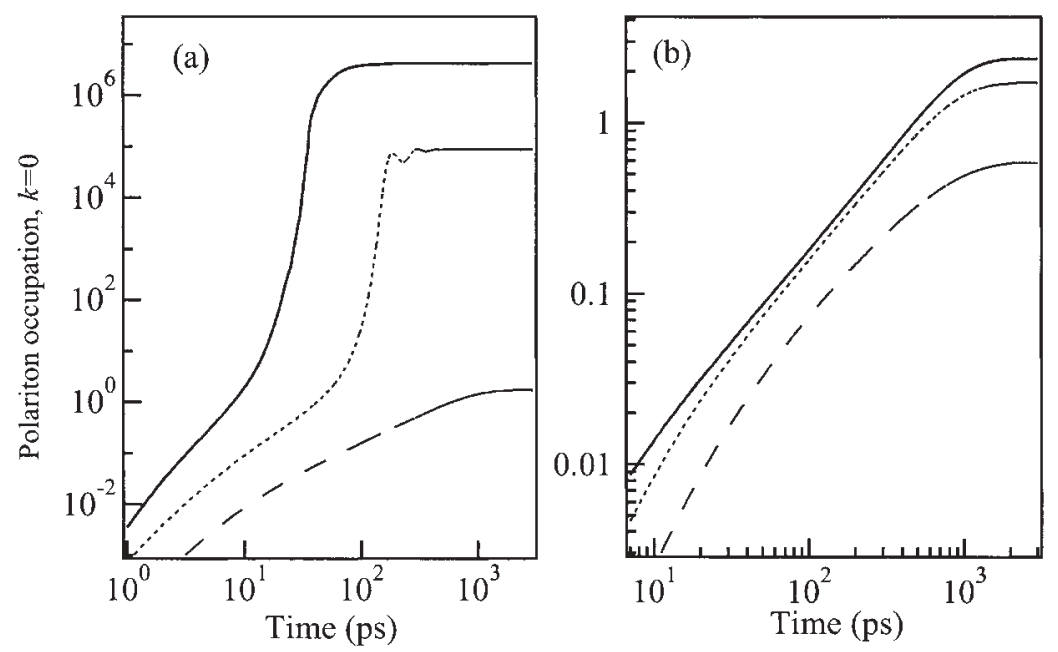

Fig. 3. a) Polariton occupation of the $k=0$ state versus time for excitation powers of $0.42 \mathrm{~W} / \mathrm{cm}^{2}$ (dashed), $4.2 \mathrm{~W} / \mathrm{cm}^{2}$ (dotted), and $168 \mathrm{~W} / \mathrm{cm}^{2}$ (solid). The pump is turned on at $t=0$. The corresponding total polariton equilibrium densities are $7.0 \times 10^{8} \mathrm{~cm}^{-2}, 1.3 \times 10^{9} \mathrm{~cm}^{-2}$, and $1.3 \times 10^{10} \mathrm{~cm}^{-2}$, respectively. b) Same as a) for a pump power of $0.42 \mathrm{~W} / \mathrm{cm}^{2}$ and electron doping of $2.5 \times 10^{9} \mathrm{~cm}^{-2}$ (dashed), $10^{10} \mathrm{~cm}^{-2}$ (dotted), and $4 \times 10^{10} \mathrm{~cm}^{-2}$ (solid)

of states that in the case of curve $b$. The equilibrium polariton density in the cavity is now $1.25 \times 10^{9} \mathrm{~cm}^{-2}$, i.e. 20 times lower than in the cases of curves a and b. The thin dotted line in Fig. 2 shows the equilibrium polariton density from a Bose distribution function plotted for zero chemical potential. The good agreement with curve (c) clearly demonstrates that a thermodynamic equilibrium is achieved for this value of the chemical potential, which is a signature of Bose condensation of polaritons.

This is confirmed by tracking the temporal evolution of the population of the ground state for different excitation powers (Fig. 3a). For the strongest pumping, the population is strongly enhanced at very short times as soon as it exceeds unity, demonstrating the Bose amplification of the final state population. The dashed curve corresponds to the threshold condition for Bose amplification, producing an equilibrium population of the $k=0$ state slightly exceeding unity, but without significant amplification occurring. For an excitation area of diameter $100 \mu \mathrm{m}$ this excitation density corresponds to an extraordinary low threshold power of $15 \mu \mathrm{W}$.

Figure $3 b$ shows the temporal evolution of the population of the ground state for different doping. The equilibrium density is strongly enhanced and exceeds the lasing threshold for electron densities above $10^{10} \mathrm{~cm}^{-2}$.

Though the calculations shown here were done for conventional GaAs-based microcavities that are presently available, they can be easily extended to future GaN-based microcavities. Once the strong coupling in GaN-cavities is achieved, they will become most suitable for studies of the polariton lasing for two basic reasons: first, experiments at room temperature should become possible, thanks to the stability of excitons in nitrides, and second, much higher levels of doping will be possible for the same reason.

Acknowledgements This work has been supported by the European Commission in the framework of the Improving of Human Potential contract CLERMONT HPRN-CT- 
1999-00132, HEFCE JR98SOBA, FMRX-CT970134, and EPSRC GR/N18598. One of us (A.D.C.) acknowledges the ONR support.

\section{References}

[1] Y. YАмамото, Nature 405, 629 (2000).

[2] F. TAssone et al., Phys. Rev. B 56, 7554 (1997).

F. TAssone et al., Phys. Rev. B 59, 10830 (1999).

[3] A.I. Tartakovskil et al., Phys. Rev. B 62, R2283 (2000).

[4] P. Sellenart et al., Phys. Rev. B 62, R16263 (2000).

[5] P. SAvvidis et al., Phys. Rev. Lett. 84, 1547 (2000).

[6] T. E. Sole, Vertical Surface Emitting Lasers, Wiley, New York 1995.

[7] Le Si Dang et al., Phys. Rev. Lett. 81, 3920 (1998).

[8] P. Senellart et al., Phys. Rev. Lett. 82, 1233 (1999).

[9] R. Huang et al., Phys. Rev. B 61, R7854 (2000).

[10] R.M. Stevenson et al., Phys. Rev. Lett. 85, 3680 (2000).

[11] C. Ciuti et al., Phys. Rev. B 62, R4825 (2000).

[12] C. Benoit à la Guillaume et al., Phys. Rev. 177, 567 (1969).

H. HAUG et al., phys. stat. sol. (b), 82, 531 (1977).

S.W. Koch et al., phys. stat. sol. (b) 89, 43 (1978).

[13] I. Galbraith et al., J. Cryst. Growth 159, 667 (1996). 